\title{
Effects of signaled shifts in water reinforcement
}

\author{
MITRI E. SHANAB, ORNA MOLAYEM, and GENE STEINHAUER \\ California State University, Fresno, California 93740
}

\begin{abstract}
The effects of introducing a compound discriminative stimulus at the time of shifting magnitude of water reward were investigated in the runway. Forty rats received either 2- or 8-sec access to water reward, and then all subjects received the larger reward. During the shift, a random half of each main group was exposed to a compound light-and-tone stimulus and the other half was not. A significant PCE was obtained only when the compound stimulus was present at the time of the shift.
\end{abstract}

The present study is one in a series of studies investigating the general hypothesis that the introduction of a discriminative stimulus (SD) at the time of the shift in reinforcement conditions would enhance the emergence of contrast (see Shanab, Molayem, Gordon, \& Steinhauer, 1981 , for a brief review of contrast). Specifically, this study deals with positive contrast as a function of upward shifts in water reinforcement when such shifts are made in either the presence or the absence of SD. In runway situations, a positive contrast effect (PCE) is said to have taken place when subjects shifted from a small to a larger reward run significantly faster than control subjects receiving the larger reward all the time. In the Skinner box, when the schedule associated with one component (varied component) is decreased while the schedule associated with the other (constant) component remains unchanged, the response rate in the latter component increases relative to its prior baseline in a direction opposite to that in the varied component. This phenomenon is called positive behavioral contrast (PBC).

The foregoing general hypothesis was derived from both the additivity theory (cf. Schwartz \& Gamzu, 1977) and neo-Hullian theory (cf. Spence, 1956). Whereas additivity theory deals specifically with positive contrast, Spence's theory can explain both positive and negative contrast effects. According to additivity theory, PBC occurs as a result of the summation of classical and operant conditioning processes. Thus, PBC occurs when the SD (constant component) becomes classically conditioned to the response following a decrease in the reinforcement schedule associated with the varied component. Since the response has already been conditioned "operantly" by the reinforcement contingency, the two classical and operant processes summate, producing an elevated response rate in the constant component. According to Spence's (1956) theory, instrumental learning is mediated by the classically formed $\mathrm{rg}_{\mathrm{g}} \mathrm{sg}$ mechanism. Specifically, Spence suggested that the subject learns to make conditioned goal responses $\left(\mathrm{r}_{\mathrm{g}}\right)$ in the goal area that, through backward generalization, are evoked at the start. The $\mathrm{r}_{\mathrm{g}}$ responses produce their own interoceptive stimuli, which become conditioned to the instrumental response. The stimuli in the alley evoke the $\mathrm{rg}_{\mathrm{g}} \mathrm{sg}$ mechanism, which not only triggers and directs the behavior of the subject but also determines the strength of the response.

Spence (1956) proposed that the $\mathrm{rg}_{\mathrm{g}} \mathrm{sg}$ was the underlying mechanism for the incentive motivation construct $(\mathrm{K})$ that combined multiplicatively with habit strength $(\mathrm{H})$ and additively with drive (D) to determine reaction potential (E). Such theorizing could explain both positive and negative contrast effects if it could be assumed that the magnitude of reward exerts a relative rather than an absolute effect on performance-in Spence's terms, if the old $\mathrm{K}$ associated with its runway stimuli (and the appropriate reward) and the new $\mathrm{K}$ associated with the SD (and the new reward) summate algebraically to produce either an increase or a decrease in response rate relative to controls (cf. Shanab \& Cavallaro, 1975).

Some support for the general hypothesis of the enhancing effects of the $\mathrm{SD}$ at the time of the shift has been obtained in two recent experiments reported by Shanab et al. (1981).

When the SD (tone) was introduced at the time of the shift in water reinforcement, only a graphical PCE was observed (Shanab et al., 1981). It is conceivable that upshifted subjects were running close to their physiological limit and thus could not surpass their controls. Moreover, it is possible that the tone was not a very salient $S^{D}$, so that it was weakly conditioned to the instrumental response. The present study addressed these two problems by shifting subjects to 8 -sec access rather than 20 -sec access to the reward, thus controlling for the presumed ceiling effect, and by introducing at the time of the shift a flashing light in addition to the tone, thus increasing the salience of the $\mathrm{SD}$. It was predicted that positive contrast would be more pronounced when the upshift was signaled by an SD than when it was made in the absence of such a stimulus.

\section{METHOD}

Design

The experiment consisted of three phases. In Phase 1 , two randomized groups consisting of 20 subjects each were assigned 
to either a 2 -sec or an 8 -sec water access condition. In Phase 2, all groups received the large reward magnitude. Each main group was divided in half, resulting in four equal groups according to a 2 by 2 factorial design. For two of the four groups, a discriminative stimulus in the form of a compound tone and light was introduced as soon as the subject broke the last photobeam of the runway; the other two groups did not receive the compound stimulus. In Phase 3, the subjects were exposed to the same conditions as in Phase 2, except that the compound stimulus appeared for the appropriate subjects as soon as each subject broke the first photobeam in the start section of the runway.

\section{Subjects}

The subjects were 40 naive male albino rats of the SpragueDawley strain about 70 days old at the beginning of the study.

\section{Apparatus}

The runway used was the same $1.5-\mathrm{m}$ runway described by Shanab et al. (1981). A series of photocells measured running speed over the start $(15 \mathrm{~cm})$, run $(71.5 \mathrm{~cm})$, and goal $(30.5 \mathrm{~cm})$ sections. In addition, two small flashing lights were installed in the Plexiglas cover, one placed in the center of the goalbox cover and the other centered in the runway cover immediately above the first set of photocells.

\section{Procedure}

Upon arrival from the supplier, the subjects were housed in individual cages with free access to food and water and were handled daily for $2 \mathrm{~min} / \mathrm{day}$ for 15 days. Subjects were then placed on a 23.75 -h water-deprivation schedule. At least $30 \mathrm{~min}$ after handling each day, the subjects were given water access for $16 \mathrm{~min}$. After a total of 14 days on the deprivation schedule, the subjects were given exploration in the runway. No water was provided in the runway during exploration. On the first 2 days, each subject was given $60 \mathrm{sec}$ of exploration of the runway with the guillotine doors open and all equipment turned off. On the 3 rd and 4 th days, the doors were operated in the same fashion as on regular trials and the equipment was turned on to accustom the subjects to its sounds. Furthermore, 2 days of operant levels were taken by measuring the time taken by each subject to traverse the entire runway on the first run. On each of these days, the subjects were given either 2- or 20-sec access to water in the goalbox $30 \mathrm{~min}$ after the completion of their trial run, in order to familiarize them with the water tube. The subjects were then assigned to two main groups, and Phase 1 was started. On the first 2 days of this phase, each subject received 1 trial/day, but 2 trials/day thereafter. On each trial, the subject was placed in the startbox and, as soon as it oriented toward the run section, both guillotine doors were opened. Interruption of the last photobeam provided each subject access to the drinking tube for either 2 or $8 \mathrm{sec}$. If a subject failed to pass through a section of the runway in $60 \mathrm{sec}$, a time of $60 \mathrm{sec}$ was recorded for that and subsequent sections of the runway. Trials were massed, with an intertrial interval of approximately $10 \mathrm{sec}$. Phase 1 lasted 10 days. The acquisition phase was made deliberately short as a further control for the ceiling effect. In Phase 2, the subjects were matched on the basis of their asymptotic performance levels in Phase 1 and all subjects received $8-\mathrm{sec}$ access to the reward. For a random half of each main group, a discriminative stimulus in the form of a mild tone (71 dB) and a flashing light was introduced as soon as the rat broke the last photobeam in the goalbox; the stimulus continued until the tube retracted $8 \mathrm{sec}$ later. Phase 2 lasted 15 days.

In Phase 3 , the $\mathrm{SD}^{\mathrm{D}}$ was introduced as soon as the rat broke the first photobeam in the startbox, and it continued until the drinking tube retracted following the 8 -sec reward period. Phase 3 lasted 22 days. This phase was introduced to see if the introduction of the $S^{D}$ at the start of the response chain would strengthen or prolong whatever contrast effect was obtained in Phase 2.

In all phases, the subjects were weighed daily before being placed in the runway and had free access to food. Moreover, during the three phases, each subject was given its 15 -min daily ration of water at least $30 \mathrm{~min}$ following its second daily trial.

\section{RESULTS}

All analyses are based on speed measures (in meters per second) over blocks of six trials each.

\section{Phase 1}

A 2 by 3 repeated-measures ANOVA test with reward magnitude as the between-subjects factor was performed over the last three blocks. A significant effect due to reward magnitude was obtained for the start and run sections of the runway $[\mathrm{F}(1,38)=8.57, \mathrm{p}<.01$, and $\mathrm{F}(1,38)=5.00, \mathrm{p}<.05$, respectively], reflecting faster running speeds for the large-reward group compared with the small-reward group. A nonsignificant reward magnitude effect was obtained for the goal section $[F(1,38)=1.08, p>.05]$, and an almost significant effect was obtained for the total section $[F(1,38)=4.94$, $p>.05]$. Neither the blocks effect nor the Magnitude by Blocks interaction effect was significant (both Fs $<1$ for all sections of the runway), suggesting that the groups had stabilized before the first shift.

\section{Phase 2}

A 2 by 2 by 4 repeated-measures ANOVA test, with the reward magnitude in Phase 1 and the stimulus conditions in Phase 2 (i.e., presence or absence of tone and light) as the between-subjects factors, was performed over Blocks 2-5.

Since the analyses of the speed measures in Phase 2 were highly similar, only the analysis of total speed is reported for this phase. Both main effects of stimulus condition and reward magnitude were found to be nonsignificant $[\mathrm{F}(1,36)=1.02, \mathrm{p}>.05$, and $\mathrm{F}(1,36)<1$, respectively]. Also, the Stimulus Condition by Magnitude interaction yielded nonsignificant results $[F(1,36)$ $=2.24, \mathrm{p}>.05]$, as did the Magnitude by Blocks interaction effect $[F(3,108)=1.03, p>.05]$. Both the Stimulus Condition by Blocks and the three-way interaction effects yielded nonsignificant results (both $\mathrm{Fs}_{S}<1$ ). The main effects of blocks yielded significant results $[F(3,108)=4.23, p<.01]$.

A test of simple effects revealed a nonsignificant PCE based on a comparison between Groups $\mathrm{SL}_{\mathrm{T}}$ and $\mathrm{LL}_{\mathrm{T}}$ for all sections of the runway [total speed: $F(1,36)=$ $2.59, p>.05]$. Similarly, a comparison of the SL and LL groups that were not exposed to the tone-and-light condition yielded nonsignificant results [total speed: $\mathrm{F}(1,36)<1]$.

\section{Phase 3}

It should be pointed out that in Phase 3 the tone and light were presented when the subjects broke the startbox photobeam and remained on until the liquid dispenser retracted. Inspection of Figure 1 reveals that in Phase 3 the differences in performance of the groups 


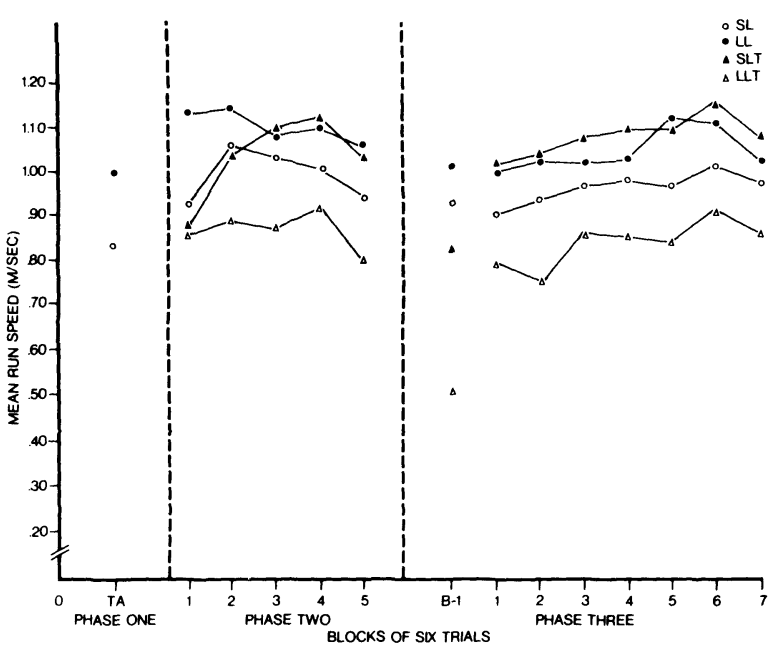

Figure 1. Mean run speed as a function of an upward shift in water reward: $S$ refers to small, $L$ to large, and $T$ to compound light and tone stimulus.

followed the same pattern as that in Phase 2 but were a little more accentuated. A graphical PCE was observed over Blocks 1-7 for the groups receiving the tone-andlight condition but not for those that did not receive the compound stimulus.

A two-way repeated-measures ANOVA test was performed over Blocks 1-7 for the start speeds, yielding a significant main effect for the blocks $[F(6,216)=8.49$, $\mathrm{p}<.001]$. Neither the main effect of magnitude nor that of the stimulus condition was significant (both Fs $<1)$. All two-way interaction effects were not significant (all Fs $<1$ ). Moreover, the Stimulus Condition by Magnitude by Blocks interaction effect was not significant $[F(6,216)=1.14, p>.05]$. Tests of simple main effects yielded nonsignificant PCEs, both when the tone and light were present $[F(1,36)=2.17, \mathrm{p}>.05]$ and when these stimuli were absent $(\mathrm{F}<1)$.

An ANOVA test of the run speeds over Blocks 2-7 yielded a significant Stimulus Condition by Magnitude interaction effect $[F(1,36)=4.11, p<.05]$. A significant blocks effect $[\mathrm{F}(5,180)=4.32, \mathrm{p}<.001]$ was also found. Neither the main effect of reward magnitude $[F(1,36)=1.03, p>.05]$ nor that of stimulus condition $(\mathrm{F}<1)$ was significant. Similarly, all other interactions were not significant (all $\mathrm{Fs}<1$ ). Tests of simple main effects revealed a significant PCE when tone and light were presented $[F(1,36)=4.46, p<.05]$, but not in the absence of this compound stimulus $(\mathrm{F}<1)$.

An ANOVA test of the goal speed over Blocks 2-7 again revealed a significant blocks effect $[F(5,180)=$ $5.48, \mathrm{p}<.01]$. Nonsignificant effects were obtained for the main effect of stimulus condition $[F(1,36)=$ $1.05, \mathrm{p}>.05]$, and the Stimulus by Magnitude $[\mathrm{F}(1,36)$ $=3.33, \mathrm{p}>.05]$, the Stimulus by Blocks $[F(5,180)=$ $1.42, \mathrm{p}>.05]$, and the Magnitude by Blocks $[\mathrm{F}(5,180)$ $<1]$ interaction effects. However, the triple interaction was significant $[F(5,180)=3.79, p<.01]$. Tests of simple effects yielded a significant PCE for the toneand-light condition $[\mathrm{F}(1,36)=4.89, \mathrm{p}<.05]$, but a nonsignificant PCE when the compound stimulus was absent $(\mathrm{F}<1)$.

The results of an ANOVA test over Blocks 2-7 of the total speed revealed only one significant effect, namely, the blocks effect $[F(5,180)=4.66, p<.01]$. All other effects were not significant, although the Stimulus Condition by Magnitude interaction effect approached significance $[F(1,36)=3.39, p<.07]$. Individual comparisons of the upshifted groups relative to the nonshifted groups yielded a significant PCE $[F(1,36)$ $=4.22, \mathrm{p}<.05]$ under tone-and-light conditions, but a nonsignificant PCE in the absence of such a compound stimulus $(F<1)$.

\section{DISCUSSION}

The results clearly show that under appropriate conditions, an upward shift in water reward leads to a significant PCE when such a shift is accompanied by $\mathrm{SD}^{\mathrm{D}}$. In this study, when the compound SD accompanied the shift in the goalbox only, a graphical, but not a statistically significant, PCE was observed. However, when the $S D$ was introduced at the initiation of the response chain and continued until the end of the chain, a highly significant PCE was obtained. Those subjects that were shifted in the absence of such SD did not show any PCE. In fact, the upshifted group apparently ran slower than its control.

That a significant PCE occurred only when the $\mathrm{SD}$ was introduced at the beginning rather than the end of the response chain is in agreement with the operant procedures observed in behavioral contrast studies. The $S^{D}$ in the operant procedure is present throughout the session. This makes the $\mathrm{SD}^{\mathrm{D}}$ a reliable predictor of the change in reinforcement conditions. To be a better predictor of the shift in reinforcement conditions in the runway, the $S^{D}$ was presented at the initiation of the response and was continued until the end of the response chain. In Spence's (1956) terms, this procedure probably not only speeded the conditioning of the $r_{g}{ }^{-s}$ mechanism but also maintained it as a constant level throughout the response chain. It is conceivable that the compound stimulus facilitated the summation of several Ks along the runway, thus augmenting the response speed.

At the empirical level, these results indicate that when an external stimulus is correlated with the upward shift in reinforcement magnitude in the runway, significant positive contrast is obtained, just as it is in the Skinner box. This effect seems to be due to an augmentation in response strength and not an artifactual result of a suppressive effect of the compound stimulus. In other words, it could be argued that the PCE obtained might be a function not merely of the elevation in response rate due to the $S^{D}$ but also of what appears as a suppressive effect of the $\mathrm{S}^{\mathrm{D}}$. It can be seen that Group $\mathrm{LL}_{T}$ ran slower than Group LL. However, despite this graphical difference, the two groups were not statistically significant in the run section over Blocks $2-7[\mathrm{~F}(1,36)=3.17, \mathrm{p}>.05]$. A similar analysis of the goal speed over Blocks 4-7 also showed a nonsignificant effect $[F(1,36)=2.86, p>.05]$. Moreover, if the notion of a suppressive effect of $S^{D}$ is correct, then a parallel difference between Groups SLT and SL should have been observed. This was not the case, for Group SL actually ran slower, not faster, than Group SLT $[F(1,36)=1.03, p>.05]$ over Blocks 2-7 of the run section.

At the theoretical level, the results are consistent with the notion that the $S^{D}$ in both the operant and instrumental situations serves as a differential predictor of the change in reinforcement conditions. According to additivity theory, the $S^{D}$ 
evokes new classically conditioned responses that add to the already operantly conditioned responses and produce the observed increase in rate of responding in the unchanged component.

According to Spence's (1956) theory, the external stimulus also augments the strength of the classically conditioned fractional anticipatory goal response, which is the underlying mechanism for incentive motivation (K). As noted earlier, relative to controls, the shifted subjects receive an additional $\mathrm{K}$ (or $\mathrm{Ks}$ ), thus accounting for the observed positive incentive contrast.

In general, the results clearly indicate that when the procedures are equated, positive contrast emerges regardless of the design or apparatus used. It is probably premature, although quite tempting, to conclude from these findings that the student of contrast does not have to look for different laws to account for the contrast effects obtained in within-subjects as opposed to between-subjects designs.

\section{REFERENCES}

Schwartz, B., \& Gamzu, E. Pavlovian control of operant: An analysis of autoshaping and its implications for operant conditioning. In W. K. Honig \& J. E. R. Staddon (Eds.), Handbook of operant behavior. Englewood Cliffs, N.J: PrenticeHall, 1977.

Shanab, M. E., \& Cavallaro, G. Positive contrast obtained in rats following a shift in schedule, delay, and magnitude of reward. Bulletin of the Psychonomic Society, 1975, 5, 109-112.

Shanab, M. E., Molayen, O., Gordon, A. C. \& Steinhauer, G. Effects of signaled shifts in liquid reinforcement. Bulletin of the Psychonomic Society, 1981, 18, 263-266.

SPEnCE, K. W. Behavior theory and conditioning. New Haven, Conn: Yale University Press, 1956.

(Received for publication January 18, 1982.) 